\title{
Three electrons in laterally coupled quantum dots: Tunnel vs electrostatic coupling, ground-state symmetry, and interdot correlations
}

\author{
B. Szafran ${ }^{1,2}$ and F. M. Peeters ${ }^{1}$ \\ ${ }^{1}$ Departement Fysica, Universiteit Antwerpen (Campus Drie Eiken), B-2610 Antwerpen, Belgium \\ ${ }^{2}$ Faculty of Physics and Applied Computer Science, AGH University of Science and Technology, al. Mickiewicza 30, \\ 30-059 Kraków, Poland
}

(Received 16 November 2004; published 21 June 2005)

\begin{abstract}
The phase diagram for the ground-state symmetry of three electrons confined in a pair of laterally coupled dots is determined as function of the interdot distance and the magnetic field. With a few exceptions the ground-state spin and parity symmetry sequence of a circular harmonic quantum dot is conserved. Reentrant behavior of some energy levels as ground states is found as a function of the magnetic field. The disappearance of interdot tunnelling due to a strong magnetic field leads to ground-state degeneracy of the even and odd parity energy levels. It is shown that at a high magnetic field the system can be closely approximated by a two-electron system confined in one dot and a spectator electron localized in the other. Broken-parity eigenstates with a classical charge distribution are constructed and used to discuss the interdot electron-electron correlations.
\end{abstract}

DOI: 10.1103/PhysRevB.71.245314

PACS number(s): 73.21.La

\section{INTRODUCTION}

Electrons confined in coupled quantum dots ${ }^{1-25}$ form systems commonly referred to as artificial molecules with single-electron wave functions forming bonding and antibonding orbitals similar to those known from the quantum chemistry of covalent molecules. Artificial molecules are formed by vertically ${ }^{1-11}$ coupled dots or by dots coupled laterally. ${ }^{12-25}$ The electronic properties of two-electron systems in vertically ${ }^{4-7}$ as well as laterally ${ }^{12-16}$ coupled dots have been extensively studied by exact methods that for two electrons are particularly convenient due to the separation of the spatial and spin degrees of freedom. The two-electron studies were mainly motivated by the proposed ${ }^{26}$ realization of a quantum gate based on the spins of the electrons confined in coupled dots. For larger electron numbers the mean field methods ${ }^{1-3,18-21}$ are more commonly used. The mean field approaches give reliable estimates for the ground-state energy and are useful in simulations 2, $3,18,19,27_{\text {of devices but }}$ they possess several shortcomings ${ }^{28}$ due to an approximate treatment of the electron-electron correlations, which results in artifactal symmetry-breaking effects leading to an oversimplified picture of Wigner crystallization, to the appearance of spin-density waves, etc.

The exact solution of the few-electron Schrödinger equation possesses a rich literature for circular two-dimensional quantum dots. ${ }^{28-39}$ Less work has been done in noncircular ${ }^{40-42}$ and vertically coupled quantum dots. ${ }^{8-11}$ In this paper we present the exact diagonalization results for the three-electron system in quantum dots coupled laterally. We are unaware of any previous exact diagonalization calculations for a pair of laterally coupled dots with more than two electrons. While in vertically coupled dots the interdot tunnelling makes the problem intrinsically three dimensional, but with conserved axial symmetry, in the laterally coupled dots the physical interest (and technical implementation difficulties) are related to the two-center nature of the electron localization.
The three-electron system in laterally coupled dots is more interesting than the extensively discussed two-electron problem ${ }^{12-16}$ for at least two reasons: (1) In the two-electron system the electron charge localized in each of the identical dots is exactly equal to $e .^{16}$ On the other hand, the threeelectron system possesses two equivalent classical configurations with two electrons in the left or right dot. We show that the three-electron parity operator eigenstates can be constructed as superpositions of the states corresponding to both classical configurations. The charge density distribution in the parity eigenstates is nonclassical; with one and one-half electron charge confined in each of the dots. Due to tunnelling each attempt to localize two electrons in one of the dots will result in the formation of a nonstationary state. The classical charge distribution can only be obtained when the tunnel coupling between the dots disappears. The previous mean field analysis of the charge distribution and symmetries dealt mostly with an even number of electrons ${ }^{20-22}$ for which the problem of nonclassical charge distribution, likely to result in an artifactal spatial symmetry breaking, is absent. (2) The evolution of the two-electron ground state ends in the singlet-triplet degeneracy ${ }^{12-16}$ when the tunnel coupling is removed by an external magnetic field. On the other hand, in the three-electron system at high-magnetic field, spin oscillations should be expected to continue in the two-electron subsystem perturbed by the Coulomb potential of the electron confined in the other dot and the electrostatic interdot coupling should $\operatorname{pin}^{43-45}$ the magnetic-field induced twoelectron Wigner molecules, extracting them from the internal coordinates of the two-electron system to the laboratory frame of reference.

In this paper we study the spin and parity symmetry of the three-electron ground state, the electron-electron correlation, the Wigner crystallization, and the extinction of tunnel coupling in the limit of high magnetic field. The high-magnetic field spectra are explained using a single-dot two-electron model, including the Coulomb potential of an electron local- 
ized in the other dot. The interdot electron-electron correlations accompanying the electrostatic interdot coupling, are studied using broken-parity eigenstates. The results presented in this paper have been obtained by the multicenter configuration interaction method described in detail in Ref. 41. This method was previously applied to problems of Wigner crystallization in low-symmetry quantum dots, ${ }^{41}$ to pinning of Wigner molecules by an external Coulomb defect, ${ }^{45}$ and to the effect of the asymmetry on the exchange energy in two-electron laterally coupled dots. ${ }^{16}$

The present paper is organized as follows. In Sec. II we present the theory, Sec. III contains the results, summary and conclusions are given in Sec. IV.

\section{THEORY}

We consider three electrons bound in a two-dimensional system of laterally coupled dots that is described by the Hamiltonian,

$$
H=\sum_{i=1}^{3} h_{i}+\sum_{i=1}^{3} \sum_{j>i}^{3} \frac{e^{2}}{4 \pi \epsilon \epsilon_{0} r_{i j}},
$$

with the single-electron energy operator $h$ defined as

$$
h=\frac{1}{2 m^{*}}(-i \hbar \boldsymbol{\nabla}+e \mathbf{A})^{2}+V(x, y),
$$

where $m^{*}$ is the electron effective band mass and $\epsilon$ is the dielectric constant. We apply the model potential for laterally coupled dots used previously by several authors, ${ }^{14,15,20,22}$

$$
V(x, y)=\frac{m^{*} \omega_{0}^{2}}{2}\left(\min \left\{(x-d / 2)^{2},(x+d / 2)^{2}\right\}+y^{2}\right),
$$

where $\hbar \omega_{0}$ is the confinement energy and $d$ is the distance between the centers of the two dots. For $d=0$ this model potential reduces to a single circular quantum dot with a harmonic oscillator confinement potential.

We use the Landau gauge $\mathbf{A}=(-B y, 0,0)$, GaAs material parameters ${ }^{14} \mathrm{~m}^{*} / \mathrm{m}_{0}=0.067, \quad \epsilon=12.4$, and choose $\hbar \omega_{0}$ $=3 \mathrm{meV}$ for the confinement potential energy. We include only the orbital effects for the ground-state spin and spatial symmetries, but we neglect the spin Zeeman effect. The latter can be trivially taken into account as a shift linear in $B$ to the calculated energy levels. At a high magnetic field the spin Zeeman effect removes the nonpolarized states from the ground-state symmetry sequence, as discussed previously. $28,37,38$

We first solve the single-electron Schrödinger equation in a basis of displaced lowest Landau level eigenfunctions $\mathrm{s}^{41,45-48}$

$$
\Psi_{\mu}(\mathbf{r})=\sum_{i=1}^{M} c_{i}^{\mu} \psi_{\mathbf{R}_{i}}(\mathbf{r})
$$

where

$$
\begin{aligned}
\psi_{\mathbf{R}_{i}}(\mathbf{r})= & \sqrt{\alpha} \exp \left\{-\alpha\left(\mathbf{r}-\mathbf{R}_{i}\right)^{2} / 4+i e B\right. \\
& \left.\times\left(x-X_{i}\right)\left(y+Y_{i}\right) / 2 \hbar\right\} / \sqrt{2 \pi},
\end{aligned}
$$

and $\mathbf{R}_{i}=\left(X_{i}, Y_{i}\right)$ is the center of localization of the $i$ th basis element. For a single quantum dot the multicenter basis (4) reproduces $^{41}$ also the Fock-Darwin eigenfunctions of the higher Fock-Darwin bands that tend to excited Landau levels at a high magnetic field. Therefore, for a single quantum dot the present scheme works as efficient as the ones employing the Fock-Darwin single electron basis. ${ }^{29-38,48}$ Since the centers can be chosen quite arbitrarily the multicenter method is suitable to treat any low-symmetry smooth confinement potential. ${ }^{41}$ In the present calculations we use 14 centers $(7$ per dot). The set of centers corresponding to the right quantum dot is chosen in the following way. A single center is localized in the point $(a, 0)$. Six additional centers are put around this point on a circle of radius $R$. The position of the centers for the left dot are obtained from the set associated with the right dot by a change of the sign of the $x$ coordinates. The basis is optimized by taking $R, a$, and $\alpha$ as variational parameters that are chosen to minimize the energy of the three-electron system.

The three-electron Hamiltonian (1) is diagonalized on the basis of Slater determinants constructed from the singleelectron spin orbitals obtained as products of the spatial wave functions expanded in the basis (4) and the eigenfunctions of the $z$ component of the single-electron spin. Eigenstates of Hamiltonian (1) are also eigenstates of the parity operator as well as of the operators of the $z$ component of the total spin (with eigenvalue $S_{z} \hbar$ ) and of the square of the total spin $\left(S(S+1) \hbar^{2}\right)$. Of all 3276 three-electron Slater determinants that can be constructed of the 28 spin orbitals we retain only those with the required $S_{z}$ and parity eigenvalues, which gives a basis of 182 basis elements for $S_{z}= \pm 3 / 2$ and 637 basis elements for $S_{z}= \pm 1 / 2$. The quantum number of the total spin $S$ is identified for each energy level by its multiplicity $(2 S+1$ - the degeneracy of the given energy level with respect to $S_{z}$ ). In the following the even (odd) symmetry states are denoted by ${ }^{S} \mathrm{E}\left({ }^{S} \mathrm{O}\right)$.

\section{RESULTS}

\section{A. Energy spectra}

Figures 1(a) and 1(b) show the low-energy spectrum of the three-electron single quantum dot $(d=0)$. We display energies calculated with respect to the lowest Fock-Darwin energy level, i.e., we subtract $3 E_{D}=3 \times \hbar \sqrt{\omega_{0}^{2}+\omega_{c}^{2} / 4}$ from the eigenvalues of Hamiltonian (1). In the absence of the magnetic field the ground state corresponds to -1 angular momentum (in $\hbar$ units). The ground-state angular momentum takes subsequent negative integer values (the absolute value of the angular momentum of the states is given in the figure) as the magnetic field increases. Ground states with angular momentum quantum numbers being multiples of 3 are realized by the spin-polarized states. ${ }^{28,34,37,49}$ At lower magnetic field the intervals corresponding to subsequent ground state symmetries have distinctly different lengths [see Fig. 1(a)]. In particular, a larger stability of the ground state with odd angular momentum quantum numbers up to -7 is observed. The results of Fig. 1(a) are in perfect agreement with the results of Mikhailov and Savostianova ${ }^{37}$ (cf. Figs. 1(a) and 2(a) of Ref. 37, $\hbar \omega_{0}=3 \mathrm{meV}$ corresponds to the interaction 

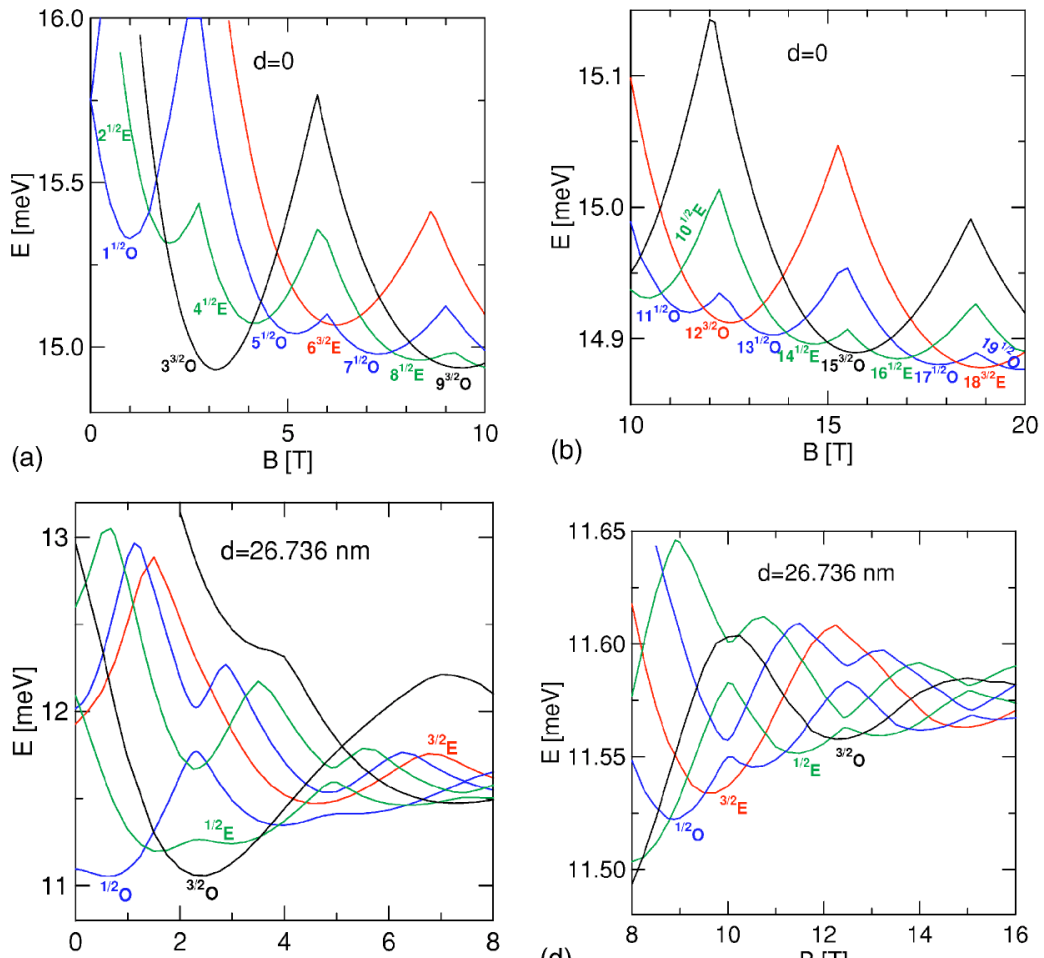

(c)
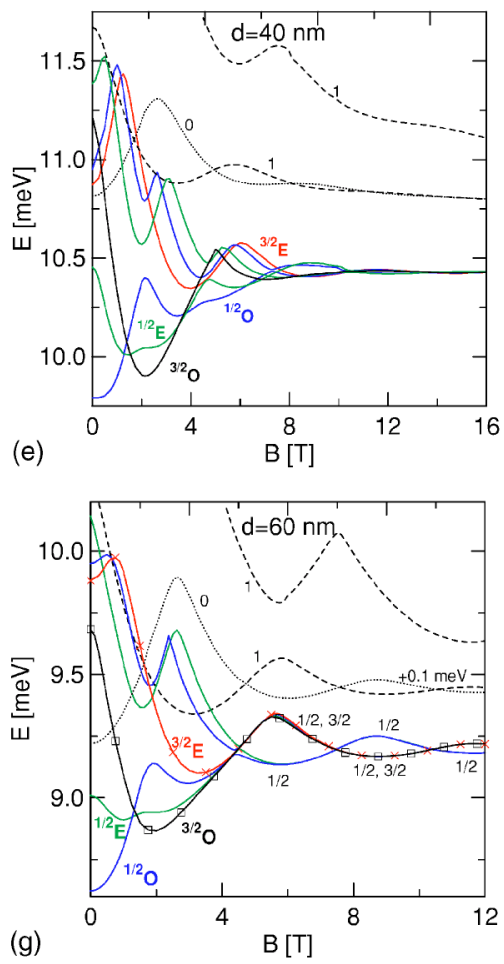
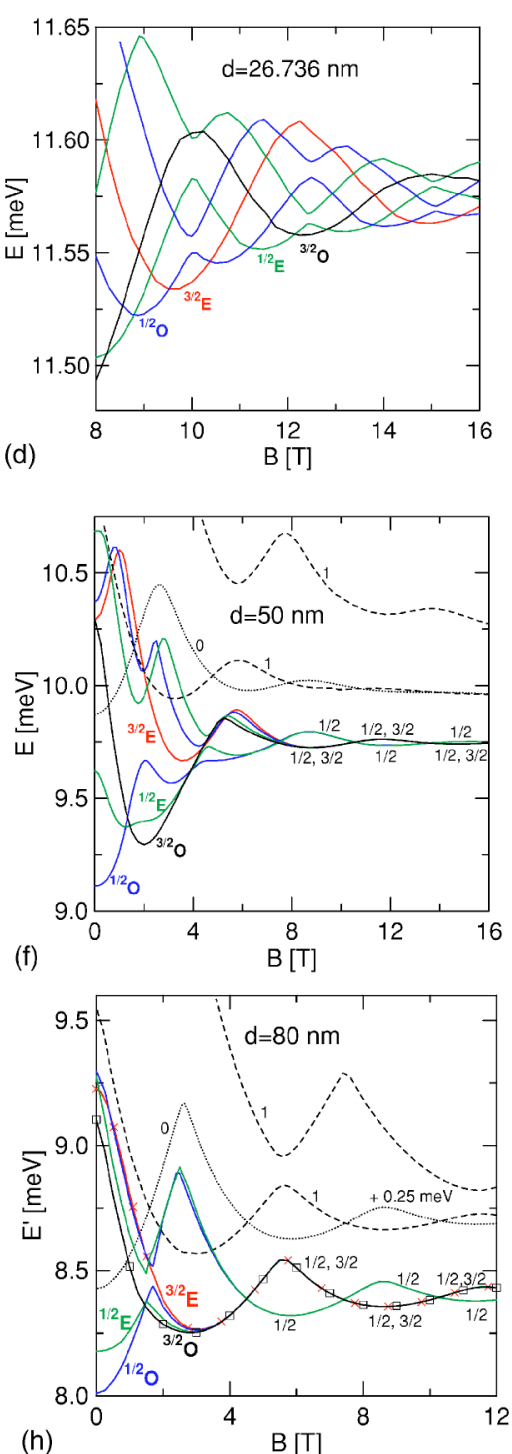

FIG. 1. (Color online) Magnetic field dependence of the three electron spectra for different values of the interdot distance $d$. Energy levels of ${ }^{1 / 2} \mathrm{O},{ }^{3 / 2} \mathrm{O},{ }^{1 / 2} \mathrm{E}$, and ${ }^{3 / 2} \mathrm{E}$ symmetry states are plotted in blue, black, green, and red, respectively. Numbers in the energy levels labels given in (a) and (b) stand for the absolute value of the angular momentum in $\hbar$ units. Dotted and dashed lines in $(\mathrm{e}-\mathrm{g})$ show the singlet and triplet energy levels in a reference two-electron system confined in the potential given by Eq. (6). Crosses and squares in $(\mathrm{g})$ and $(\mathrm{h})$ mark the energy levels of spin-polarized states of even and odd parity, respectively. Numbers $0,1,1 / 2$, and $3 / 2$ in Figs. $1(\mathrm{e})-1(\mathrm{~h})$ give the spin quantum number of the plotted energy levels. For clarity, the two-electron spectrum in $(\mathrm{g})$ and $(\mathrm{h})$ was shifted by +0.1 and $+0.25 \mathrm{meV}$, respectively. parameter $\lambda=1.988)$. At higher fields [cf. Fig. 1(b)] the states with increasing angular momentum become the ground state in intervals of nearly constant length in magnetic field. A similar feature has previously been observed in the spinpolarized three-electron system in the Wigner crystallization regime. ${ }^{48}$

Figures 1(c) and 1(d) present the spectrum for coupled dots with centers separated by a distance of $d=26.736 \mathrm{~nm}$. In a single circular dot energy levels of the same spin and parity symmetry differ by their angular momentum and cross. Notice that in the coupled dots these crossings are replaced by anticrossings. Apparent crossings of ${ }^{3 / 2} \mathrm{O}$ and ${ }^{1 / 2} \mathrm{O}$ energy levels around 5.5 and $7.5 \mathrm{~T}$ visible in Fig. 1(c) are in fact anticrossings of width $2 \mu \mathrm{eV}$. The spectrum conserves the same sequence of the ground-state spin and parity symmetries of the single dot case [cf. Figs. 1(a) and 1(b)]. The only difference is that the ${ }^{3 / 2} \mathrm{E}$ state (the ground state for $d=0$ around $5.6 \mathrm{~T}$ ) is replaced by the ${ }^{1 / 2} \mathrm{O}$ energy level stemming 
from the mixture of states which in circular quantum dots possess -5 and -7 angular momenta. The ${ }^{1 / 2} \mathrm{O}$ energy level is particularly stable as the ground state [cf. Fig. 1(a)] and exhibits reentrant behavior. At higher magnetic field [see Fig. $1(d)]$ the ground-state changes almost periodically with $B$ like in the single dot case presented in Fig. 1(b), only the length of the magnetic field corresponding to subsequent states of the sequence becomes shortened from about 1.1 to $0.9 \mathrm{~T}$. At high magnetic field the energy differences between the energy levels of different parity becomes smaller with respect to the single dot case presented in Fig. 1(b). For both $d=0$ and $d=26.736 \mathrm{~nm}$ the energy difference between the lowest even and odd spin-polarized energy levels possesses a local maximum near $B=12 \mathrm{~T}$. For $d=0$ this difference is around $0.25 \mathrm{meV}$ [cf. Fig. 1(b)] while for $d$ $=26.736 \mathrm{~nm}$ it is only $0.05 \mathrm{meV}$ [cf. Fig. 1(d)].

Figure 1(e) shows the spectrum for $d=40 \mathrm{~nm}$. For $B$ $<10 \mathrm{~T}$ the ground-state-symmetry sequence of the $d$ $=26.736 \mathrm{~nm}$ case is reproduced. Near 8.5 T both the spinpolarized and spin-nonpolarized energy levels become degenerate. For larger barrier thickness the ground state becomes degenerate with respect to parity around 7, 6 and $4 \mathrm{~T}$, for $d=50,60$, and $60 \mathrm{~nm}$, respectively, [see Figs. 1(f)-1(h)]. The magnetic field leads to an increase of the electron localization in each of the dots, enhancing the effective barrier height and leading finally to vanishing interdot tunnel coupling. This is at the origin of the even-odd degeneracy, similarly as in the single electron problem.

For an interdot separation of $d=40 \mathrm{~nm}$, the low-energy spectrum collapses into a narrow energy range for large magnetic fields. For $B>12 \mathrm{~T}$ the difference between the lowest energy levels becomes smaller than $0.02 \mathrm{meV}$ [see Fig. 1(e)]. But, for a larger interdot distance we notice, e.g., for $d$ $=50 \mathrm{~nm}$ [cf. Fig. 1(f)] above $8 \mathrm{~T}$ distinct spin-related oscillations of the ground-state energy. Up to $10.5 \mathrm{~T}$ and between 13.5 and $17 \mathrm{~T}$ the two spin-polarized ground states of odd and even symmetry are degenerate with two nonpolarized states of both spatial symmetries. Between 10.5 and $13.5 \mathrm{~T}$ the ground state is nonpolarized. The amplitude of these spin oscillations decreases with magnetic field but increases with interdot distance. For instance, the local maximum of the energy splitting between the lowest spin-polarized and nonpolarized energy levels near $12 \mathrm{~T}$ is $0.03,0.04$, and $0.06 \mathrm{meV}$ for $d=50,60$, [see Fig. $1(\mathrm{~g})$ ] and $80 \mathrm{~nm}$ [see Fig. $1(\mathrm{~h})]$, respectively.

\section{B. Two-electron subsystem and a spectator electron in the other dot}

One may expect that in the absence of a tunnel effect, i.e., for larger $d$ and high magnetic field, the system can be reduced to a spectator electron sitting in one dot and two electrons confined in the other dot. In order to verify this hypothesis, we have performed two-electron calculations for a single dot with harmonic oscillator confinement potential perturbed by the Coulomb potential of the electron sitting in the other (left) dot, namely, for the external potential we took

$$
V_{s}(x, y)=\frac{m^{*} \omega_{0}^{2}}{2}(x-d / 2)^{2}+\frac{e^{2}}{4 \pi \epsilon \epsilon_{0}} \frac{1}{\sqrt{(x+d / 2)^{2}+y^{2}}} .
$$

Obviously, in the presence of the Coulomb interdot coupling the spectator electron will be shifted off the center of the left quantum dot, but we neglect this shift for simplicity. Two electron calculations were performed with the multicenter configuration interaction method with eight centers put on a circumference of an ellipse and the ninth one in its center. Position of the ellipse center as well as its $x$ and $y$ sizes are optimized variationally. In Figs. 1(e)-1(h) the lowest singlet energy level and the two lowest triplet energy levels are plotted with dotted and dashed lines, respectively. The width of the presented avoided crossings of the spin polarized energy levels can be considered as a measure of the potential deviation from circularity; as discussed in Ref. 45. In the circular quantum dot limit of infinite $d$, the avoided crossings are replaced by crossings of the magic angular momenta eigenstates corresponding to the semiclassical Wigner distribution of electrons in the inner coordinates. The larger the deviation of the potential from circularity the stronger is the mixing of the states corresponding to neighbor angular momenta from the magic sequence, and, consequently, the wider the anticrossing. ${ }^{45}$ The width of the anticrossing appearing near $6 \mathrm{~T}$ decreases from $0.7 \mathrm{meV}$ for $d=40 \mathrm{~nm}$ to $0.3 \mathrm{meV}$ for $d=60 \mathrm{~nm}$ and to $0.2 \mathrm{meV}$ for $d=80 \mathrm{~nm}$. A comparison of Figs. 1(e)-1(h) shows that the singlet-triplet oscillations in the two-electron system have the smallest amplitude for the smallest $d$ for which the perturbation of the harmonic potential is the largest. This finding is consistent with the recent study $^{52}$ of the magnetic field effect on the two-electron anisotropic quantum dots showing that the amplitude of the singlet-triplet oscillations ${ }^{53}$ disappears with increasing degree of asymmetry and is finally replaced by the singlettriplet degeneracy in the quasi one-dimensional limit $[50,51]$ of the extreme anisotropy. In circular quantum dots the states of higher angular momentum are less strongly localized. Magnetic field increases the electron localization and consequently the electron-electron interaction in each of the states. This leads to the ground-state angular momentum transitions. For two electrons the ground-state of the center-of-mass corresponds to zero angular momentum so that the entire angular momentum is carried by the relative electron-electron motion. The relative-motion states with odd (even) parity angular momentum quantum numbers are spin triplets (singlets). Therefore, the increase of the angular momentum is accompanied by singlet-triplet oscillations. ${ }^{53}$ Magnetic field evolution is different for strongly anisotropic quantum dots in which the two electrons occupy the opposite extremities of the quantum dot potential and the external magnetic field simply increases the localization of each of the electrons leading eventually to the disappearance of the overlap of their wave functions which results in the singlet-triplet degeneracy $^{54}$ (vanishing exchange energy). As the interdot distance $d$ increases, the potential becomes more circular which is the reason why the amplitude of the singlet-triplet energy oscillations becomes larger. ${ }^{52}$

The three-electron spectra presented in Figs. 1(e)-1(h) display a striking similarity to the reference two-electron calculations at a high magnetic field. Therefore, at high magnetic field the system is indeed separable into two subsystems confined in different dots. The single electron confined in one of the dots does not participate in the magnetic field evolution of the spectrum and its only role is to perturb the circular symmetry of the confinement potential 


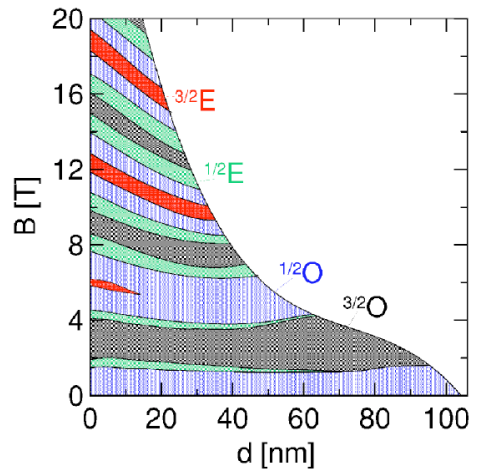

FIG. 2. (Color online) Magnetic field-interdot distance phase diagram for the ground-state symmetry. Blue, green, black, and red regions correspond to a ground state with ${ }^{1 / 2} \mathrm{O},{ }^{1 / 2} \mathrm{E},{ }^{3 / 2} \mathrm{O}$, and ${ }^{3 / 2} \mathrm{E}$ symmetry, respectively. The white region corresponds to a negligible interdot tunnel coupling and a near degeneracy of the ground state with respect to spatial parity symmetry.

felt by the two electrons in the other dot. Obviously the three-electron system can be spin polarized only if the twoelectron subsystem is spin polarized, which explains the spin dependence of the three-electron low-energy spectrum at a high field [cf. Fig. 1(e)-1(h)]. The discussed spin oscillations in the three-electron system have a somewhat larger amplitude that is due to an exaggerated anisotropy of the external potential in the two-electron reference calculation. The point charge assumed in potential (6) deforms the quantum dot circular potential more strongly than the real charge of the spectator electron, which is in fact diffuse and displaced to the left (see below) from the center of the left dot.

\section{Phase diagram for the ground-state symmetry}

Our results for the ground-state symmetry and the extinction of the interdot tunnel coupling is summarized in a $d-B$ phase diagram presented in Fig. 2. The ${ }^{3 / 2} \mathrm{E}$ ground state around $6 \mathrm{~T}$ disappears for $d>14 \mathrm{~nm}$. Similarly, the ground state of ${ }^{1 / 2} \mathrm{E}$ symmetry around $2 \mathrm{~T}$ located between the ${ }^{1 / 2} \mathrm{O}$ and ${ }^{3 / 2} \mathrm{O}$ ground states disappears above $d>70 \mathrm{~nm}$. The border of the white region of the phase diagram corresponds to negligible tunnel interdot coupling and was determined as the line beyond which the energy difference between the ground state and the lowest excited state of the same spin but opposite spatial parity is smaller than $0.02 \mathrm{meV}$.

\section{Charge density evolution}

The evolution of the ground-state charge density as a function of the external magnetic field is presented in Fig. 3 for $d=26.736 \mathrm{~nm}$. The charge density exhibits two maxima near $x= \pm 28 \mathrm{~nm}$. In each of the subsequent ground states, localization of electrons in the left and right dots becomes stronger. For low magnetic fields the shape of the charge density is similar to the one of the three-electron ellipsoidal quantum dot (cf. Fig. 4 in Ref. 41). Nevertheless, at higher magnetic field the three-electron charge density in the ellipsoidal dot develops two maxima along the $y$ axis $^{41}$ related to the position of one of the electrons in the two classically degenerate configurations. Figure 3 shows a different behavior: the electron charge density is removed from the $y$ axis at which the barrier potential energy is maximal. Note that in the state ${ }^{3 / 2} \mathrm{E}$ at $5 \mathrm{~T}$ [cf. Fig. 3(1)] the central hole in the charge density is larger than in the ground states for the neighboring range of magnetic fields [cf. Figs. 3(e)-3(h)].

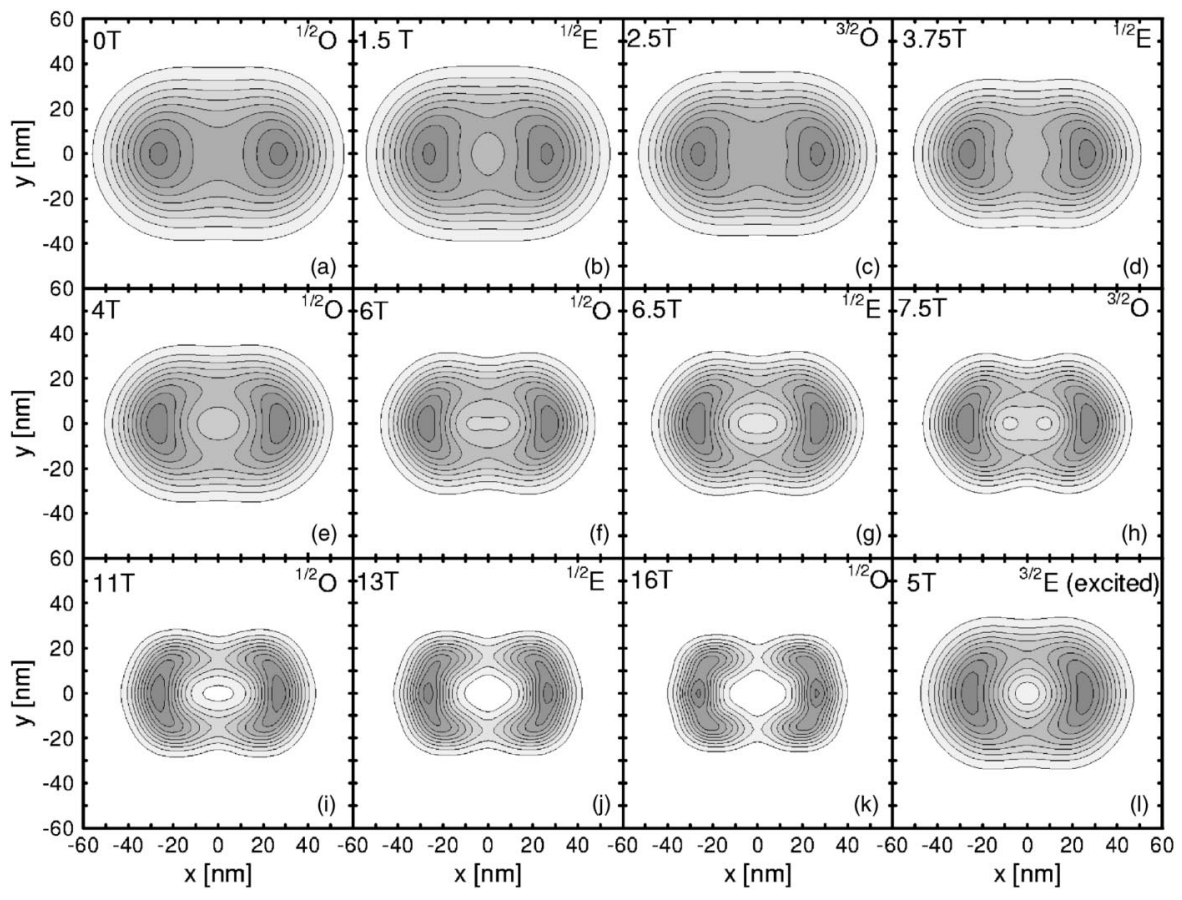

FIG. 3. Contour plots of the charge density for $d=26.736 \mathrm{~nm}$ and various magnetic fields. The plots (a-k) correspond to the ground state. Plot (1) at $B=5 \mathrm{~T}$ corresponds to an excited state of ${ }^{3 / 2} \mathrm{E}$ symmetry. 


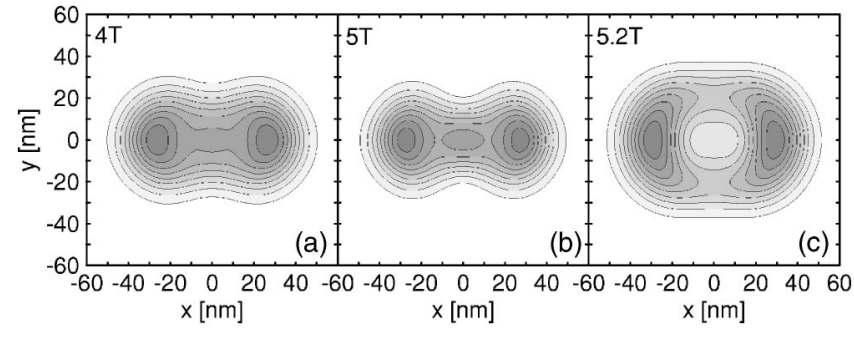

FIG. 4. Charge density contours for the lowest-energy state of ${ }^{3 / 2} \mathrm{O}$ symmetry for various values of the magnetic field and $d$ $=26.736 \mathrm{~nm}$.

This is the reason why this symmetry is replaced by the ${ }^{1 / 2} \mathrm{O}$ symmetry [cf. Figs. 1(c) and 2] in the ground state.

Increasing the magnetic field leads to an increased electron localization in each of the states. For a given magnetic field the subsequent states in the ground-state symmetry sequence correspond to weaker electron localization. In order to keep the interelectron distances approximately constant as the magnetic field increases, the system has to change the symmetry of the ground state. The mechanism for groundstate symmetry transformations is therefore the same as the one in circular quantum dots (see Sec. III B). Between the avoided level crossings, the increased localization in each of the states induced by increasing the magnetic field is usually not accompanied by any pronounced quantitative change of the shape of the charge density droplet. An exception to this rule is observed for the ${ }^{3 / 2} \mathrm{O}$ state. Figure 4 shows the charge density of this state for magnetic field values for which this state is no longer the ground state. For $4 \mathrm{~T}$ a "bridge" of an increased density along the $x$ axis appears [compare Fig. 3(c) and Fig. 4(a)]. Just before the narrow avoided crossing near $5.1 \mathrm{~T}$ [cf. Fig. 1(d)], a third charge density maximum is formed in the barrier around the origin [see Fig. 4(b)]. In this way the system forms a quasi-one-dimensional Wigner molecule ${ }^{50,51,54}$ with all charge maxima situated along the $x$ axis. Recently, it was proven ${ }^{54}$ that the spatial parity of the spin polarized three-electron state must be odd in order to allow the state to form such a one-dimensional Wigner molecule. After the anticrossing of spin polarized odd parity energy levels near $5.1 \mathrm{~T}$, the lowest state of this symmetry possesses a central charge density hole [cf. Fig. 4(c)]. The shape of the charge density of this state, when it becomes the ground state, is displayed in Fig. 3(h).

Let us now look at the magnetic-field dependence of the electron-electron correlations. Figure 5 shows the contour plots for the pair correlation function ${ }^{39}$ when the position of one of the electrons is fixed at the point $(-28 \mathrm{~nm}, 0)$. For $B<6 \mathrm{~T}$ the two other electrons are simply shifted to the right-hand side of the double quantum dot potential. Only for $B>6 \mathrm{~T}$ the two distinct centers of electron localization in the right quantum dot appear. Wigner localization around these centers, which coincide with the position of classical electrons ${ }^{55}$ in the lowest-energy configuration, ${ }^{41}$ becomes more pronounced at higher magnetic fields. Fig. 5(1) shows the excited ${ }^{3 / 2} \mathrm{E}$ energy level. Besides a larger central charge density hole [see Fig. 3(1)] this state exhibits also a stronger electron-electron correlation than the ground state [cf. Figs. $5(\mathrm{e})$ and $5(\mathrm{f})]$ in this magnetic field range.

\section{E. Broken-parity solutions and interdot correlations}

For the parity operator eigenstates discussed so far one cannot tell in which of the dots the two electrons are localized. Let us now consider construction ${ }^{41}$ of the stationary states with a classical charge density distribution with two electrons confined in a specified left or right dot.

In single quantum dots the classical degeneracy, ${ }^{41}$ i.e., the existence of more than one classical lowest-energy configuration of electrons, is accompanied by crossings of levels of different symmetries. Superpositions of the two states corresponding to the crossing levels allows us to extract ${ }^{41}$ one of the classical configurations. Here we use a similar manipulation, constructing a superposition of odd $(O)$ and even $(E)$ parity wave functions,

$$
X=(E+\exp (i \phi) O) / \sqrt{2} .
$$

The state described by the wave function $X$ is not an eigenstate of the parity operator but at the even-odd degeneracy points appearing at the level crossings, or for negligible tunnel coupling, it is still stationary, i.e., it is still the energy eigenstate.

For $d=26.736 \mathrm{~nm}$ near $B=8.75 \mathrm{~T}$, level crossings of odd and even parity energy levels appear [cf. Fig. 1(d)] for both $S=1 / 2$ and $S=3 / 2$ states. We use this degeneracy to illustrate the properties of the broken-parity Hamiltonian eigenstates. Figure 6 shows the charge accumulated on the righthand side of the $y=0$ axis as a function of the phase $\phi$ in Eq. (7). Notice that in the singlet broken-parity state the right dot can contain up to 1.64 of the elementary charge. For the triplet the maximum value is $1.89 e$. The maximum value of the charge localized in the right dot reaches $2 e$ (see the curve for $d=80 \mathrm{~nm}$ ) only when the tunnel coupling is completely removed by the application of a high magnetic field and/or for a large barrier thickness.

The charge density of the degenerate-energy spinpolarized states at $B=8.75 \mathrm{~T}$ is displayed in Fig. 7(a) (the even parity state) and Fig. 7(b) (the odd parity state). Figure 7(c) shows the broken-parity state constructed of the two spin-polarized parity eigenstates for the phase in Eq. (7) for which the charge localized at the $x>0$ semiplane is maximal [cf. Fig. 6]. Figure 7(c) shows that in spite of the leakage of the two-electron charge through the barrier to the region of negative $x$, the charge of the third electron is distinctly separated. Such a separation is not visible in the singlet state plotted in Fig. 7(d). According to Eq. (7) both odd and even parity states can be reconstructed from two broken-parity states with two electrons localized in the right and left dot. This is visible in the pair-correlation function plots presented in Figs. 7(e) and 7(f) for the odd-parity spin-polarized state. Depending on the choice of the fixed-electron position a configuration with two electrons in the left [cf. Fig. 7(e)] or right dot [cf. Fig. 7(f)] appears in the pair-correlation function plots. This property of the parity eigenstates makes it difficult to visualize the interdot correlations between the electron positions: instead of the reaction of the charge localized in one dot to the position of an electron in the other, switching between configurations is observed. However, the effect of interdot correlations can be conveniently visualized using 


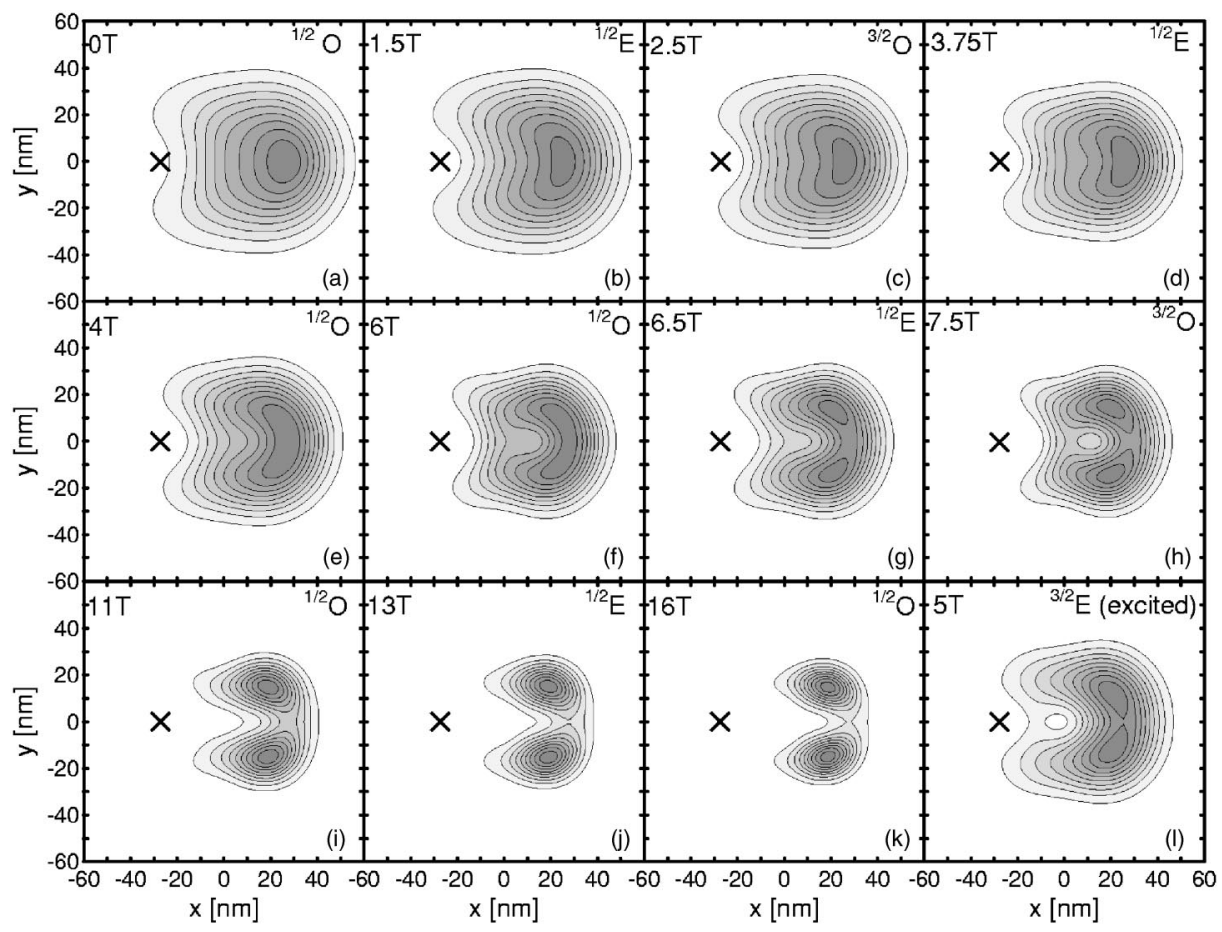

FIG. 5. Pair correlation functions for $d=26.736 \mathrm{~nm}$ and various magnetic fields. One of the electrons is fixed at the position $(-28 \mathrm{~nm}, 0)$ (indicated by the cross). All the plots (a-k) with the exception of the plot (1) for the state ${ }^{3 / 2} \mathrm{E}$ at $B=5 \mathrm{~T}$ correspond to the ground state.

the pair correlation plots for the broken-parity states [cf. Figs. $7(\mathrm{~g})$ and $7(\mathrm{~h})$ ]. For the electron position fixed in the center of the left dot [see Fig. 7(h)], the two other electrons are more firmly localized in the right dot than in the odd parity state [Fig. 7(f)]. In the broken-parity state, a displacement of the fixed position electron to the position of one of the two charge maxima occupied in the configuration with two electrons in the left dot induces a rotation of the electrons in the right dot [cf. Fig. $7(\mathrm{~g})]$. Note that due to this rotation the electron charge localized in the lower part of the right dot tunnels through the barrier to the left of the $y=0$ axis. For $d=26.736$ and $B=8.75 \mathrm{~T}$ the tunnel coupling between the dots is still not negligible (see Fig. 2).

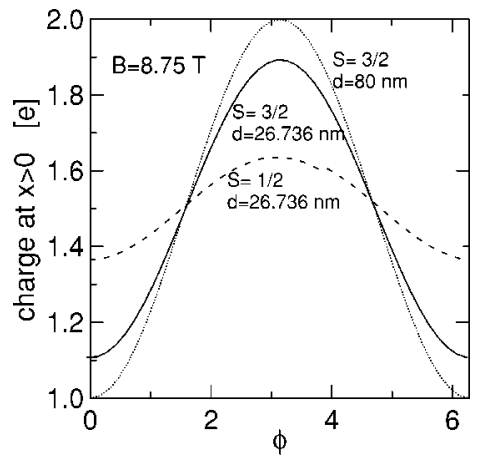

FIG. 6. Charge accumulated in the right quantum dot for the broken-parity Hamiltonian eigenstates as function of the phase of the superposition [Eq. (7)] for $S=1 / 2$ (dashed line) and $S=3 / 2$ (solid line) at $d=26.736 \mathrm{~nm}$ and for the spin-polarized state at $d$ $=80 \mathrm{~nm}$ (dotted line).
Let us now look at a spin-polarized broken-parity state for $d=40 \mathrm{~nm}$ and $B=8.4 \mathrm{~T}$, i.e., in the neighborhood of the border of the phase diagram of Fig. 2, beyond which tunnel coupling is negligible. This specific value of the magnetic field has been chosen because it corresponds to a crossing of spin polarized even and odd energy levels. Although the tunnelling of the electrons from the right to the left dot is not visible in the charge density plot [Fig. 8(a)] the pair correlation plot [Fig. 8(c)] shows that it is not totally absent. Fig. 8(b) shows the lowest triplet state for the reference twoelectron calculation with potential (6). The separation of the charges of the two electrons occupying the right quantum dot in the reference calculation is stronger than in the threeelectron plot of Fig. 8(a).

Figure 9 shows the results for $d=60 \mathrm{~nm}$ and $B=8.5 \mathrm{~T}$ deep inside the region of vanishing tunnel coupling on the phase diagram presented in Fig. 2. The ellipsoidal deformation of the charge confined in the left dot is visibly smaller than in the preceding plots [see Figs. 7(c) and 8(a)]. The charge density confined in the right quantum dot [Fig. 9(a)] is more similar to the reference two-electron calculations [Fig. 9(b)] than for $d=40 \mathrm{~nm}$. No tunnel coupling is observed either in the charge density or in the pair correlation plots. After the disappearance of the tunnel effect, the Coulomb coupling of the charge in both dots is still accompanied by quantum interdot correlations in the electron positions [compare Figs. 9(c) and 9(d)].

For $d=80 \mathrm{~nm}$ and $B=8.5 \mathrm{~T}$, the charge density in the right quantum dot [Fig. 10(a)] becomes identical to the twoelectron quantum dot perturbed by an external Coulomb potential [Fig. 10(b)]. The same shape is also reproduced by the pair correlation function for an electron placed in the left dot 


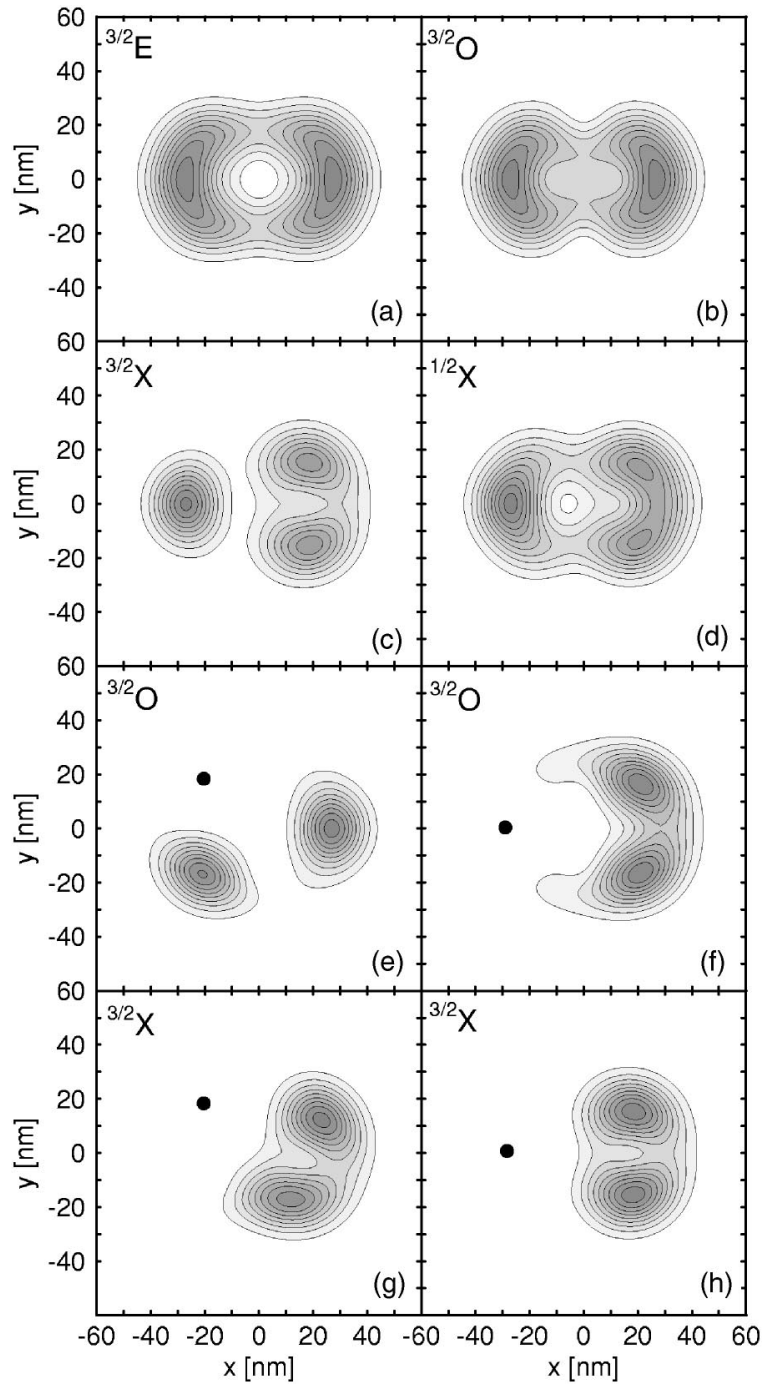

FIG. 7. Charge density (a-d) and pair correlation function (e-h) plots for $d=26.736 \mathrm{~nm}$ at $B=8.75 \mathrm{~T}$. Plots (a), (b), (e), (f) correspond to parity eigenstates and (c), (d), (g), (h) to Hamiltonian eigenstates of a broken parity. In (e) and (g) [(f) and (h)] an electron is fixed at $(-28 \mathrm{~nm}, 0),[(-19 \mathrm{~nm}, 19 \mathrm{~nm})]$. The fixed electron position in $(\mathrm{e}-\mathrm{h})$ is marked by a dot.

[Fig. 10(c)]. The distribution of the pair correlation function is now independent of the position of the electron in the left dot, which signifies that the interdot electron-electron correlations are nonexistent. The electrons in different dots does not react any longer on their actual positions, so that the wave function of the system can be exactly separated into a product of wave functions for each of the dots like in the Hartree approximation. Figure 10 shows that the potential of the charge confined in the left dot pins the Wigner molecule induced by the external magnetic field in the right quantum dot. Perturbation of the two-electron spectra for the Coulomb center in the left dot is still strong [cf. the wide anticrossings of the triplet energy levels in Fig. 1(h)]. For larger values of $d$ the Wigner molecule pinning acquires reentrant ${ }^{45}$ character for which the laboratory-frame electron separation is distinctly more pronounced for magnetic fields corresponding to the level anticrossings.

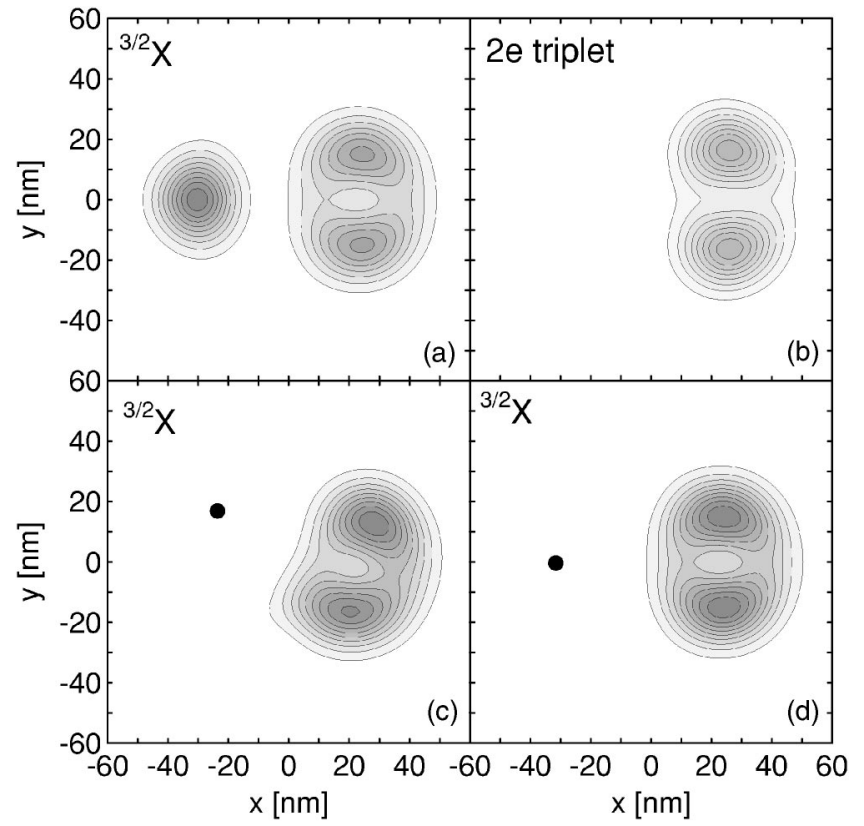

FIG. 8. Charge density (a) and pair correlation functions (c, d) plots for $d=40 \mathrm{~nm}$ at $B=8.4 \mathrm{~T}$. Charge density of the two-electron spin polarized state in a single dot with a point repulsive center at $40 \mathrm{~nm}$ from the center of the dot plotted in (b). In (c) and (d) an electron is fixed (indicated by the dot) at $(-23 \mathrm{~nm}, 16 \mathrm{~nm})$ and at $(-31 \mathrm{~nm}, 0)$, respectively.

\section{SUMMARY AND CONCLUSIONS}

We have studied the three-electron system confined in laterally coupled dots in an external perpendicular magnetic

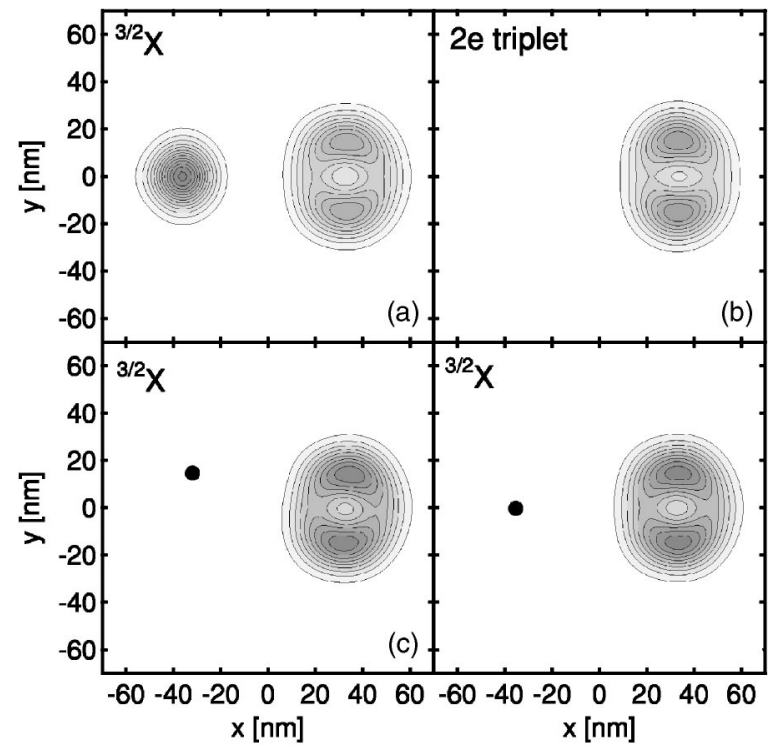

FIG. 9. Charge density (a) and pair correlation functions (c, d) plots of the spin-polarized broken-parity state for $d=60 \mathrm{~nm}$ at $B$ $=8.5 \mathrm{~T}$. Charge density of the two-electron spin-polarized state in a single dot with a point repulsive center at $60 \mathrm{~nm}$ from the center of the dot plotted in (b). In (c) and (d) an electron is fixed at ( -32 , $15 \mathrm{~nm})$ and at $(-36 \mathrm{~nm}, 0)$, respectively. The electron position is marked by a dot. 


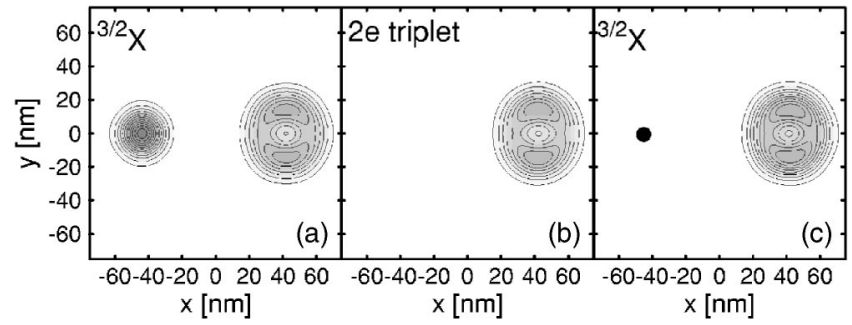

FIG. 10. Charge density (a) and pair correlation functions (c) plots for the spin-polarized broken-parity state for $d=80 \mathrm{~nm}$ at $B$ $=8.5 \mathrm{~T}$. Charge density of the two-electron spin polarized state in a single dot with a point repulsive center at $80 \mathrm{~nm}$ from the center of the dot plotted in (b). In (c) an electron is fixed at $(-44$, $0 \mathrm{~nm}$ )— marked by a dot.

field. The phase diagram for the spatial and spin parities of the ground state as function of the interdot distance and external magnetic field was determined. Near degeneracy of the ground state with respect to parity was used as a criterion for the disappearance of the tunnel coupling between the dots occurring for large interdot distances and/or at high magnetic fields. The three-electron system in laterally coupled dots reproduces the ground-state spin and parity symmetries of circular quantum dots. The exception to this rule is the absence of the spin-polarized even parity ground state which in circular quantum dots has angular momentum $-6 \hbar$ and which in laterally coupled dots turns out to be overcorrelated, i.e., correlated more strongly than the ground state. After the disappearance of tunnel coupling the spectrum exhibits spin oscillations that can be described using a model of a two-electron quantum dot perturbed by the Coulomb potential of a spectator electron localized in the other dot. We have shown that interdot electron correlations are present after the extinction of the tunnel coupling. For larger interdot distances quantum interdot correlation disappears although electrostatic interdot coupling is still significant. The effect of the Coulomb interdot coupling for the singly occupied dot is trivial, leading to a shift of the single-electron charge off the dot's center. On the other hand the Coulomb coupling induces pinning of the magnetic-field induced two-electron Wigner molecules, i.e., their extraction from the internal coordinates of the two-electron system to the laboratory frame of reference.

\section{ACKNOWLEDGMENTS}

This paper was partly supported by the Flemish Science Foundation (FWO-V1), the Belgian Science Policy, the University of Antwerpen (VIS and GOA). BS is supported by the EC Marie Curie IEF Project No. MEIF-CT-2004-500157.
${ }^{1}$ B. Partoens and F. M. Peeters, Phys. Rev. Lett. 84, 4433 (2000).

${ }^{2}$ M. Pi, A. Emperador, M. Barranco, F. Garcias, K. Muraki, S. Tarucha, and D. G. Austing, Phys. Rev. Lett. 87, 066801 (2001).

${ }^{3}$ D. G. Austing, S. Tarucha, H. Tamura, K. Muraki, F. Ancilotto, M. Barranco, A. Emperador, R. Mayol, and M. Pi, Phys. Rev. B 70, 045324 (2004).

${ }^{4}$ G. Burkard, G. Seelig, and D. Loss, Phys. Rev. B 62, 2581 (2000).

${ }^{5}$ S. Bednarek, T. Chwiej, J. Adamowski, and B. Szafran, Phys. Rev. B 67, 205316 (2003).

${ }^{6}$ D. Bellucci, M. Rontani, F. Troiani, G. Goldoni, and E. Molinari, Phys. Rev. B 69, 201308(R) (2004).

${ }^{7}$ B. Partoens, A. Matulis, and F. M. Peeters, Phys. Rev. B 59, 1617 (1999).

${ }^{8}$ J. J. Palacios and P. Hawrylak, Phys. Rev. B 51, 1769 (1995).

${ }^{9}$ S. C. Benjamin and N. F. Johnson, Phys. Rev. B 51, 14733 (1995).

${ }^{10}$ H. Imamura, P. A. Maksym, and H. Aoki, Phys. Rev. B 53, 12613 (1996).

${ }^{11}$ M. Rontani, S. Amaha, K. Muraki, F. Manghi, E. Molinari, S. Tarucha, and D. G. Austing, Phys. Rev. B 69, 085327 (2004).

${ }^{12}$ G. Burkard, D. Loss, and D. P. DiVincenzo, Phys. Rev. B 59, 2070 (1999).

${ }^{13}$ X. Hu and S. Das Sarma, Phys. Rev. A 61, 062301 (2000).

${ }^{14}$ A. Harju, S. Siljamäki, and R. M. Nieminen, Phys. Rev. Lett. 88, 226804 (2002).

${ }^{15}$ M. Marlo, A. Harju, and R. M. Nieminen, Phys. Rev. Lett. 91, 187401 (2003).

${ }^{16}$ B. Szafran, F. M. Peeters, and S. Bednarek, Phys. Rev. B 70,
205318 (2004)

${ }^{17}$ C. Yannouleas and U. Landman, Phys. Rev. Lett. 82, 5325 (1999).

${ }^{18}$ S. Nagaraja, J.-P. Leburton, and R. M. Martin, Phys. Rev. B 60, 8759 (1999).

${ }^{19}$ R. Ravishankar, P. Matagne, J. P. Leburton, R. M. Martin, and S. Tarucha, Phys. Rev. B 69, 035326 (2004).

${ }^{20}$ A. Wensauer, O. Steffens, M. Suhrke, and U. Rössler, Phys. Rev. B 62, 2605 (2000).

${ }^{21}$ J. Kolehmainen, S. M. Reimann, M. Koskinen, and M. Manninen, Eur. Phys. J. B 13, 731 (2000).

${ }^{22}$ C. Yannouleas and U. Landman, Eur. Phys. J. D 16, 373 (2001).

${ }^{23}$ F. R. Waugh, M. J. Berry, D. J. Mar, R. M. Westervelt, K. L. Campman, and A. C. Gossard, Phys. Rev. Lett. 75, 705 (1995).

${ }^{24}$ A. W. Holleitner, C. R. Decker, H. Qin, K. Eberl, and R. H. Blick, Phys. Rev. Lett. 87, 256802 (2001).

${ }^{25}$ J. M. Elzerman, R. Hanson, J. S. Greidanus, L. H. Willems van Beveren, S. De. Franceschi, L. M. K. Vandersypen, S. Tarucha, and L. P. Kouwenhoven, Phys. Rev. B 67, 161308(R) (2003).

${ }^{26}$ D. Loss and D. P. DiVincenzo, Phys. Rev. A 57, 120 (1998).

${ }^{27}$ S. Bednarek, B. Szafran, and J. Adamowski, Phys. Rev. B 65, 035316 (2001)

${ }^{28}$ S. M. Reimann and M. Manninen, Rev. Mod. Phys. 74, 1283 (2002).

${ }^{29}$ P. A. Maksym and T. Chakraborty, Phys. Rev. B 45, R1947 (1992).

${ }^{30}$ P. Hawrylak, and D. Pfannkuche, Phys. Rev. Lett. 70, 485 (1993).

${ }^{31}$ S. R. Eric Yang, A. H. MacDonald, and M. D. Johnson, Phys. Rev. Lett. 71, 3194 (1993). 
${ }^{32}$ J. J. Palacios, L. Martin-Moreno, G. Chiappe, E. Louis, and C. Tejedor, Phys. Rev. B 50, R5760 (1994).

${ }^{33}$ M. Eto, Jpn. J. Appl. Phys., Part 1 36, 3924 (1997).

${ }^{34}$ P. A. Maksym, Phys. Rev. B 53, 10871 (1996).

${ }^{35}$ A. Wójs and P. Hawrylak, Phys. Rev. B 56, 13227 (1997).

${ }^{36}$ M. Manninen, S. Viefers, M. Koskinen, and S. M. Reimann, Phys. Rev. B 64, 245322 (2001).

${ }^{37}$ S. A. Mikhailov and N. A. Savostianova, Phys. Rev. B 66, 033307 (2002).

${ }^{38}$ M. B. Tavernier, E. Anisimovas, F. M. Peeters, B. Szafran, J. Adamowski, and S. Bednarek, Phys. Rev. B 68, 205305 (2003).

${ }^{39}$ P. A. Maksym, H. Immamura, G. P. Mallon, and H. Aoki, J. Phys.: Condens. Matter 12, R299 (2000).

${ }^{40}$ M. Manninen, M. Koskinen, S. M. Reimann, and B. Mottelson, Eur. Phys. J. D 16, 381 (2001).

${ }^{41}$ B. Szafran, F. M. Peeters, S. Bednarek, and J. Adamowski, Phys. Rev. B 69, 125344 (2004).

${ }^{42}$ A. Harju, E. Räsanen, H. Saarikoski, M. J. Puska, R. M. Nieminen, and K. Niemelä, Phys. Rev. B 69, 153101 (2004).

${ }^{43}$ B. Reusch and R. Egger, Europhys. Lett. 64, 84 (2003).
${ }^{44}$ A. D. Güçlü, J.-S. Wang, and H. Guo, Phys. Rev. B 68, 035304 (2003).

${ }^{45}$ B. Szafran and F. M. Peeters, Europhys. Lett. 66, 701 (2004).

${ }^{46}$ C. Yannouleas and U. Landman, Phys. Rev. B 66, 115315 (2002).

${ }^{47}$ J. Kainz, S. A. Mikhailov, A. Wensauer, and U. Rösser, Phys. Rev. B 65, 115305 (2002).

${ }^{48}$ B. Szafran, S. Bednarek, J. Adamowski, M. B. Tavernier, E. Anisimovas, and F. M. Peeters, Eur. Phys. J. D 28, 373 (2004).

${ }^{49}$ C. G. Bao, W. Y. Ruan, and Y. Y. Liu, Phys. Rev. B 53, 10820 (1996).

${ }^{50}$ K. Jauregui, W. Häusler, and B. Kramer, Europhys. Lett. 24, 581 (1993).

${ }^{51}$ W. Häusler and B. Kramer, Phys. Rev. B 47, 16353 (1993).

${ }^{52}$ P. S. Drouvelis, P. Schmelcher, and F. K. Diakonos, Phys. Rev. B 69, 155312 (2004).

${ }^{53}$ M. Wagner, U. Merkt, and A. V. Chaplik, Phys. Rev. B 45, R1951 (1992).

${ }^{54}$ B. Szafran, F. M. Peeters, S. Bednarek, T. Chwiej, and J. Adamowski, Phys. Rev. B 70, 035401 (2004).

${ }^{55}$ V. M. Bedanov and F. M. Peeters, Phys. Rev. B 49, 2667 (1994). 